The Astrophysical Journal, 418:L45-L48, 1993 November 20

(C) 1993. The American Astronomical Society. All rights reserved. Printed in U.S.A.

\title{
CORONAL ABUNDANCES OF NEON AND MAGNESIUM ISOTOPES FROM SOLAR ENERGETIC PARTICLES
}

\author{
R. S. Selesnick, A. C. Cummings, J. R. Cummings, R. A. Leske, R. A. Mewaldt, and E. C. Stone \\ California Institute of Technology, Pasadena, CA 91125 \\ AND \\ T. T. VON RosENVINGE \\ NASA/Goddard Space Flight Center, Greenbelt, MD 20771 \\ Received 1993 July 14; accepted 1993 September 1
}

\begin{abstract}
The relative abundances of 22 to $70 \mathrm{MeV}$ nucleon $^{-1} \mathrm{Ne}$ and $\mathrm{Mg}$ isotopes from two large flare-associated solar energetic particle (SEP) events in 1992 October and November, are determined from data taken by the Mass Spectrometer Telescope on the polar-orbiting SAMPEX satellite. After converting the measured abundances to coronal values, the average ${ }^{22} \mathrm{Ne} /{ }^{20} \mathrm{Ne}$ ratio is $0.076 \pm 0.008$, consistent with the solar wind value of 0.073 but significantly less than that of previous SEP measurements, in which the abundance of ${ }^{22} \mathrm{Ne}$ was substantially enhanced. The average ${ }^{26} \mathrm{Mg} /{ }^{24} \mathrm{Mg}$ ratio is $0.152 \pm 0.012$, consistent with both previous SEP and other solar system measurements.

Subject headings: Sun: abundances - Sun: corona - Sun: flares
\end{abstract}

\section{INTRODUCTION}

Studies of the elemental composition of the solar energetic particles (SEPs), in conjunction with spectroscopic and solar wind measurements, have proved useful in determining the corresponding abundances in the solar corona and photosphere (Breneman \& Stone 1985; Meyer 1985; review by Stone 1989). They also provide insight into the transport and acceleration processes that must occur between the photosphere, corona, and the interplanetary observation site (e.g., von Steiger \& Geiss 1989; Meyer 1992). Flare-to-flare variations in the SEP elemental composition are to a large extent ordered by the ionic charge-to-mass ratio (Breneman \& Stone 1985). By correcting for the fractionation effects of acceleration and transport, Breneman \& Stone (1985) were able to determine coronal abundances for elements with atomic number $Z=6-28$. Similar studies of SEP isotopic abundances have been restricted to only a few elements and have relatively large uncertainties (Mewaldt \& Stone 1989), owing to the experimental difficulty in resolving isotopes of a given element and to the relatively poor statistical accuracy achieved in measuring the rare isotopes of a given element. The launch of the $S A M P E X$ satellite into a polar orbit in 1992 July, provided an opportunity to address these issues with the Mass Spectrometer Telescope (MAST), designed for high-resolution, large geometry factor measurements of isotopes with nuclear charge from 2 to 30 ( $\mathrm{He}$ to $\mathrm{Ni}$ ) in the energy range from $\sim 15$ to $\sim 200$ $\mathrm{MeV}$ nucleon $^{-1}$.

The isotopes of neon have proved to be of particular interest in studies of solar system composition (see, e.g., Podosek 1978) because of the high degree of variability in the abundance ratio ${ }^{22} \mathrm{Ne} /{ }^{20} \mathrm{Ne}$ from different sources. The SEP ${ }^{22} \mathrm{Ne} /{ }^{20} \mathrm{Ne}$ ratio has been measured previously by summing data over a number of flare events (Dietrich \& Simpson 1979; Simpson, Wefel, \& Zamow 1983) and in the large 1978 September 23 solar event (Mewaldt et al. 1979; Mewaldt, Spalding, \& Stone 1984; Mewaldt \& Stone 1989). The results were consistent with the ratio $\sim 0.122$ observed in the meteoric componant $\mathrm{Ne}-\mathrm{A}$ and apparently inconsistent with the solar wind value $0.073 \pm 0.02$ measured on Apollo (Geiss et al. 1972). In addition, there is evidence for SEP nuclei implanted in lunar soil with a ${ }^{22} \mathrm{Ne}$ / ${ }^{20} \mathrm{Ne}$ ratio significantly greater than that in the solar wind (e.g., Black 1983). It thus appeared as if the Sun produces two distinct isotopic components depending on the energy range of observation, presumably because of fractionation effects in the acceleration of one or both components. Because the 1978 September 23 event was the only individual solar event for which a significant number of ${ }^{22} \mathrm{Ne}$ were detected, it was not possible to adequately address the issue of possible flare-toflare variations in these earlier data sets. However, in this Letter we show that the SEP ${ }^{22} \mathrm{Ne} /{ }^{20} \mathrm{Ne}$ ratios determined from two large events observed by MAST on 1992 October and November 2 (Selesnick et al. 1993) had values near that of the solar wind and significantly less than previous spacecraft SEP measurements. We contrast this result with the abundance ratio ${ }^{26} \mathrm{Mg} /{ }^{24} \mathrm{Mg}$, for which the available SEP measurements and other solar system values are in general agreement.

\section{INSTRUMENT AND DATA ANALYSIS}

MAST consists of an array of silicon solid state detectors of graduated thickness, including four position-sensitive devices that form an X-Y hodoscope for determination of the path length through each detector triggered by a given particle event (Cook et al. 1993). The energy losses in each triggered detector are combined with a range-energy relationship to determine the particle's nuclear charge, mass, and incident energy. We have currently considered only those events stopping in one of the three detectors following the hodoscope (D1 to D3), for which there are from five to seven independent energy loss measurements, and further restricted the isotopes of each element to a common energy-per-nucleon interval.

The nuclear charge, or atomic number, $Z$, of a given event is estimated by a least-squares fit to the energy losses in each detector, weighted by their standard deviations for all events of that type, assuming that the nuclear mass $M=2 Z$. The integer nuclear charge values of each particle event are virtually unambiguous because the nature of the range-energy relationship 
results in the energy losses being relatively insensitive to the errors in the assumed $M$ values. The actual $M$ values are then estimated by a second least-squares fit for each event in which $Z$ is fixed at the nearest integer to the value determined by the first fit. Background events are eliminated by requiring that the reduced $\chi^{2}$ of the second fit satisfy $\chi^{2}<2$, resulting in the elimination of $\sim 25 \%$ of all events classified as having a given $Z$. For normally distributed data without background, $\sim 8 \%$ of all events would be eliminated by this technique. One source of background is chance coincidences in the front detectors of heavy ions $(Z>2)$ with $\gtrsim 1 \mathrm{MeV}$ protons during periods of high proton flux, resulting in larger than expected energy deposits and inaccurate particle trajectories for the heavy ions of interest. The isotopic abundance ratios were found to be insensitive to the maximum allowed value of the reduced $\chi^{2}$ over a substantial range.

In order to avoid contamination by geomagnetically trapped heavy ions (Cummings et al. 1993), only events observed at invariant latitudes $\Lambda>55^{\circ}$ are included in the analysis. To correct for the mass-dependent selection effect of the geomagnetic cutoff, the observed abundance ratios are multiplied by a factor, derived from the Størmer theory for dipole magnetic fields (e.g., Rossi \& Olbert 1970), of $C=\theta / \sin ^{-1}\left(\sin \theta\left[M_{2} / M_{1}\right]^{1 / 4}\right)$. Here $M_{2} / M_{1}$ is the corresponding mass ratio, and $\theta$ is the nominal cutoff colatitude $\left(\sim 30^{\circ}\right)$ for each element. These corrections are small; $C=0.974$ for ${ }^{22} \mathrm{Ne} /{ }^{20} \mathrm{Ne}$, for example, so that the simple approximation should be adequate.

A second factor is applied to convert the abundances to coronal values using the approach of Breneman \& Stone (1985). They found that fractionation effects due to acceleration and propagation are well described by the function $(Q / M)^{\alpha}$, where $Q / M$ is the ionic charge-to-mass ratio and $\alpha$ varies from flare to flare. Mewaldt \& Stone (1989) suggested that the measured $\mathrm{Fe} / \mathrm{O}$ ratio in a flare, $(\mathrm{Fe} / \mathrm{O})_{f}$, provides a reasonable estimate for $\alpha$ because the typical $Q / M$ ratios of these two abundant elements differ by almost a factor of 2 , with average charge states of $Q_{\mathrm{Fe}}=14.9 \pm 0.09$ and $Q_{\mathrm{o}}=7.00$ \pm 0.02 that do not appear to vary significantly from flare to flare in large SEP events (Luhn et al. 1984). Following Mewaldt \& Stone (1989) we use

$$
\alpha=\frac{\ln \left[(\mathrm{Fe} / \mathrm{O})_{f} /(\mathrm{Fe} / \mathrm{O})_{c}\right]}{\left.\ln \left[(Q / M)_{\mathrm{Fe}} / Q / M\right)_{\mathrm{o}}\right]},
$$

with the recent coronal $\mathrm{Fe} / \mathrm{O}$ ratio of $(\mathrm{Fe} / \mathrm{O})_{c}=0.172 \pm 0.025$ (Garrard \& Stone 1993) in place of the value 0.224 used by Mewaldt \& Stone. The coronal abundance ratio of two isotopes $i$ and $j$ is then given by

$$
\frac{S_{i}}{S_{j}}=\left(\frac{M_{i}}{M_{j}}\right)^{\alpha}\left(\frac{N_{i}}{N_{j}}\right) .
$$

where $N_{i} / N_{j}$ is the observed abundance ratio and $M_{i} / M_{j}$ is the corresponding mass ratio of the two isotopes. Preliminary elemental compositions from MAST data for the 1992 solar events give $(\mathrm{Fe} / \mathrm{O})_{f}$ ratios of $0.031 \pm 0.007$ for the first event and $0.071 \pm 0.006$ for the second event, which lead to multiplicative conversion factors of, for example, $1.39 \pm 0.06$ and $1.18 \pm 0.015$, respectively, for ${ }^{22} \mathrm{Ne} /{ }^{20} \mathrm{Ne}$, and $1.32 \pm 0.05$ and $1.15 \pm 0.015$, respectively, for ${ }^{26} \mathrm{Mg} /{ }^{24} \mathrm{Mg}$. These conversion factors are slightly larger than those given in a previous report (Selesnick et al. 1993) due to a revision of the $(\mathrm{Fe} / \mathrm{O})_{f}$ values. They are relatively insensitive to our choice of the Garrard \&

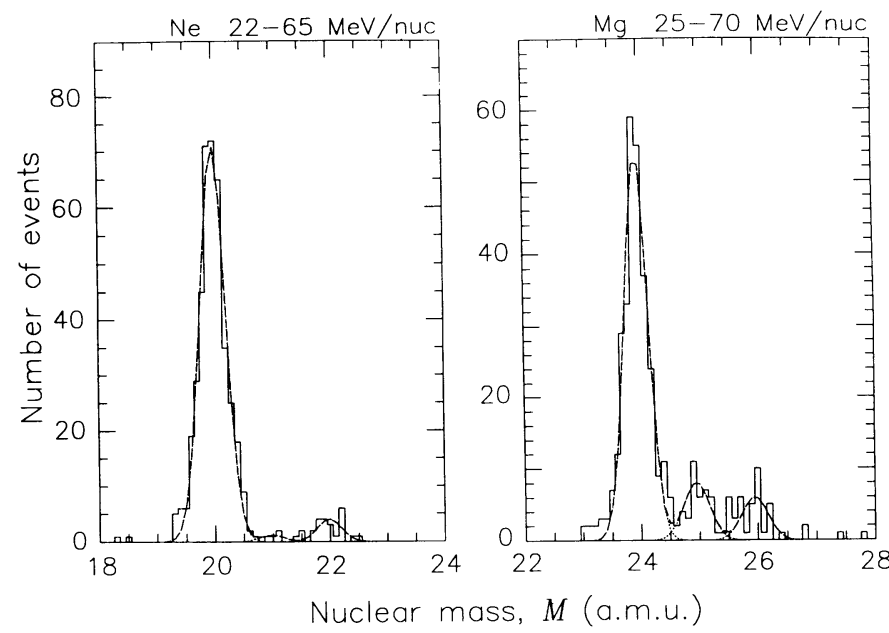

FIG. 1.-Mass histograms and Gaussian fits for $\mathrm{Ne}(22$ to $65 \mathrm{MeV}$ nucleon $\left.^{-1}\right)$ and $\mathrm{Mg}\left(25\right.$ to $\left.70 \mathrm{MeV}^{\text {nucleon }}{ }^{-1}\right)$ from the 1992 October 30 solar particle event.

Stone $(\mathrm{Fe} / \mathrm{O})_{c}$ value, varying by $<2 \%$ were we to use either $(\mathrm{Fe} / \mathrm{O})_{c}=0.19$ based on X-ray observations (Parkinson 1977) or 0.159 based on solar wind data (Bochsler 1989). Similarly, an uncertainty of $10 \%$ in the value of $(\mathrm{Fe} / \mathrm{O})_{f}$ translates to an uncertainty of only $\sim 2 \%$ in the resulting coronal value of ${ }^{22} \mathrm{Ne} /{ }^{20} \mathrm{Ne}$.

\section{RESULTS}

Histograms of the estimated $M$ for $\mathrm{Ne}(Z=10)$ and $\mathrm{Mg}$ $(Z=12)$ are shown for each of the two large SEP events in Figures 1 and 2, respectively. Gaussian fits to the mass histograms are included for the stable isotopes. Referring to Figures 1 and 2 , rare isotopes such as ${ }^{22} \mathrm{Ne}$ that are separated from the more abundant isotope by 2 amu are clearly resolved, and nearly identical abundance ratios are obtained from the Gaussian fit and from the total number of events within \pm 0.5 amu of each integer value of $M$. While resolved peaks are also visible in the histograms for isotopes separated from the abundant isotopes by $1 \mathrm{amu}$, the Gaussian model may be inaccurate in these cases, so that the abundance ratios derived by this method should be considered only as preliminary estimates.

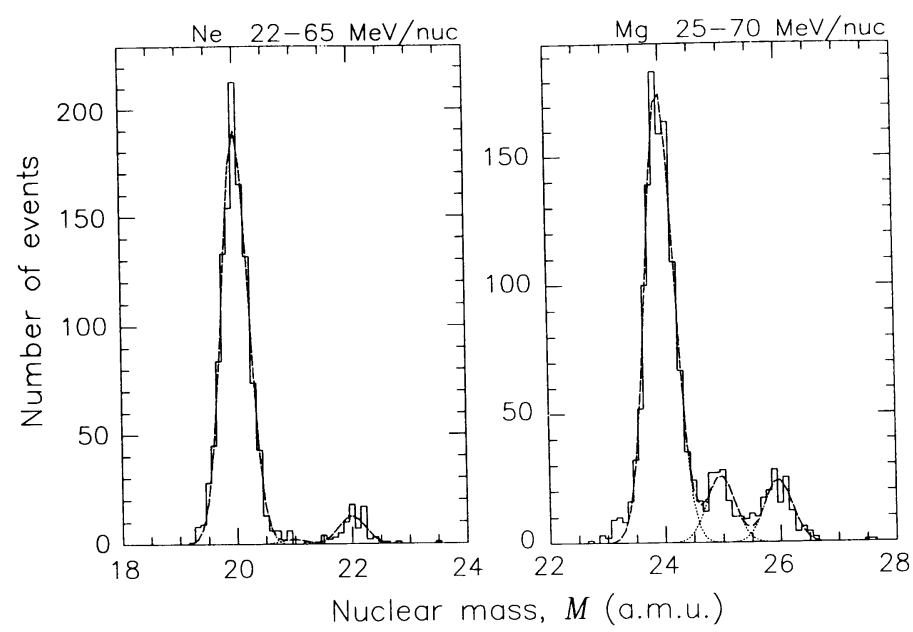

FIG. 2.-Same as Fig. 1 but for the 1992 November 2 solar particle event 
TABLE 1

CORONAL Isotopic Abundance Ratios

\begin{tabular}{lcccccc}
\hline \hline & $\begin{array}{c}\text { Energy Interval } \\
\left(\text { MeV nucleon }^{-1}\right)\end{array}$ & $\begin{array}{c}\text { Flare 1 } \\
(1992 \text { Oct 10) }\end{array}$ & $\begin{array}{c}\text { Flare 2 } \\
(1992 \text { Nov 2) }\end{array}$ & Flare 1 + Flare 2 & $\begin{array}{c}\text { 1978 September 23 } \\
\text { Flare }\end{array}$ & “Solar System "a \\
\hline${ }^{21} \mathrm{Ne} /{ }^{20} \mathrm{Ne} \ldots \ldots$. & $22-65$ & $(<0.027)$ & $(<0.016)$ & $(<0.017)$ & $<0.015$ & 0.0024 \\
${ }^{22} \mathrm{Ne} /{ }^{20} \mathrm{Ne} \ldots \ldots$. & $22-65$ & $0.0724 \pm 0.0165$ & $0.0772 \pm 0.0094$ & $0.0760 \pm 0.0081$ & $0.125_{-0.021}^{+0.029}$ & 0.073 \\
${ }^{25} \mathrm{Mg} /{ }^{24} \mathrm{Mg} \ldots \ldots$ & $25-70$ & $(0.186 \pm 0.030)$ & $(0.152 \pm 0.013)$ & $(0.157 \pm 0.012)$ & $0.157_{-0.27}^{+0.049}$ & 0.126 \\
${ }^{26} \mathrm{Mg} /{ }^{24} \mathrm{Mg} \ldots \ldots$ & $25-70$ & $0.157 \pm 0.029$ & $0.151 \pm 0.013$ & $0.152 \pm 0.012$ & $0.167_{-0.029}^{+0.048}$ & 0.139 \\
\hline
\end{tabular}

NoTE.-Preliminary coronal isotopic abundance ratios with energy-per-nucleon intervals for the 1992 flare events. Values in parentheses are subject to possible systematic uncertainties, as described in the text. Flare $1+$ Flare 2 refers to the combination of the two flare events.

a Anders \& Ebihara 1982.

More accurate modeling should reduce the abundances of these rare isotopes due to the non-Gaussian "tails" on the histograms of the abundant isotopes ${ }^{20} \mathrm{Ne}$ and ${ }^{24} \mathrm{Mg}$.

For each of the two SEP events, coronal isotopic abundance ratios with $1 \sigma$ uncertainties are listed in Table 1 . Statistical uncertainties in the $(\mathrm{Fe} / \mathrm{O})_{f}$ ratio, which contribute through the measured-to-coronal conversion factors, are included but are dominated by statistical uncertainties in the measured isotopic abundance ratios. The ratios apply to equal energy-pernuclean intervals for each isotope of a given element, as listed in the table. Since the isotopic abundances from the two flare events were not significantly different, average abundance ratios for the two events combined are also listed in Table 1.

\section{DISCUSSION}

Each of the two SEP events was associated with a major solar flare. A class X1.7/2B flare began at 1816 UT on October 30 from the location $25^{\circ} \mathrm{S}, 66^{\circ} \mathrm{W}$. A class X9.0 X-ray flare began at 0308 UT on November 2 from the same region as the October 30 flare, which was then just beyond the west limb of the Sun. The associated flux increases observed by MAST began near 1910 and 0435 UT on October 30 and November 2, respectively. These gradual-type SEP events, also known as major proton events, are generally thought to result from particle acceleration at the shock driven by a coronal mass ejection (CME) (e.g., Kahler 1993). Both of the solar flares were associated with large CMEs near the west limb of the Sun (the data on flare and CME location are from the NOAA Daily Summaries of Solar Geophysical Activity). A distinct class of interplanetary SEP events is associated with impulsive flares and includes ${ }^{3} \mathrm{He}$ rich events (Reames 1990). These are generally smaller than the gradual events and often have anomalously high $\mathrm{Fe} / \mathrm{O}$ ratios. The events observed by MAST were clearly large, gradual events.

A summary of the ${ }^{22} \mathrm{Ne} /{ }^{20} \mathrm{Ne}$ ratio for the two 1992 flare events and a comparison with previous measurements is shown in Figure 3, adapted from Mewaldt \& Stone (1989). The previous SEP data, corrected to coronal abundances by Mewaldt \& Stone (1989), have a further small adjustment for Garrard \& Stone's (1993) coronal Fe/O value of 0.172 . The data from the 1978 September 23 flare event were also associated with a large, gradual solar flare with an $\mathrm{Fe} / \mathrm{O}$ ratio of $\sim 0.08$. However, our measured ${ }^{22} \mathrm{Ne} /{ }^{20} \mathrm{Ne}$ ratios from the 1992 flares are significantly $(\sim 2 \sigma)$ lower. Although the data from the 1978 September 23 event extended to lower energies, the ${ }^{22} \mathrm{Ne} /{ }^{20} \mathrm{Ne}$ ratio from that event does not change significantly when the data are restricted to the energy range of the 1992 results (Mewaldt, Spalding, \& Stone 1984). The sevenflare average measurement from Dietrich \& Simpson (1979) included some Fe-rich SEP events, but the statistical accuracy in the individual flare events was insufficient to identify possible flare-to-flare variations. Although the measured four-flare average value of $0.06_{-0.02}^{+0.04}$ from Simpson, Wefel, \& Zamow (1983) led them to suggest the possibility of flare-to-flare variations, Figure 3 indicates that the resulting coronal value of $0.08_{-0.03}^{+0.06}$ for this flare average does not differ significantly from any of the other available measurements as a result of its large statistical uncertainty.

The 1992 flare ${ }^{22} \mathrm{Ne} /{ }^{20} \mathrm{Ne}$ ratios are close to the solar wind value of $0.073 \pm 0.002$ (Geiss et al. 1972), adopted by Anders \& Ebihara (1982) for their table of solar system abundances (abundance ratios from Anders \& Ebihara and from the 1978 September 23 SEP event are included in Table 1 for comparison with our coronal values). Previous flare measurements were closer to the value of ${ }^{22} \mathrm{Ne} /{ }^{20} \mathrm{Ne}=0.122$ for the meteoritic component $\mathrm{Ne}-\mathrm{A}$, adopted as the solar system standard by

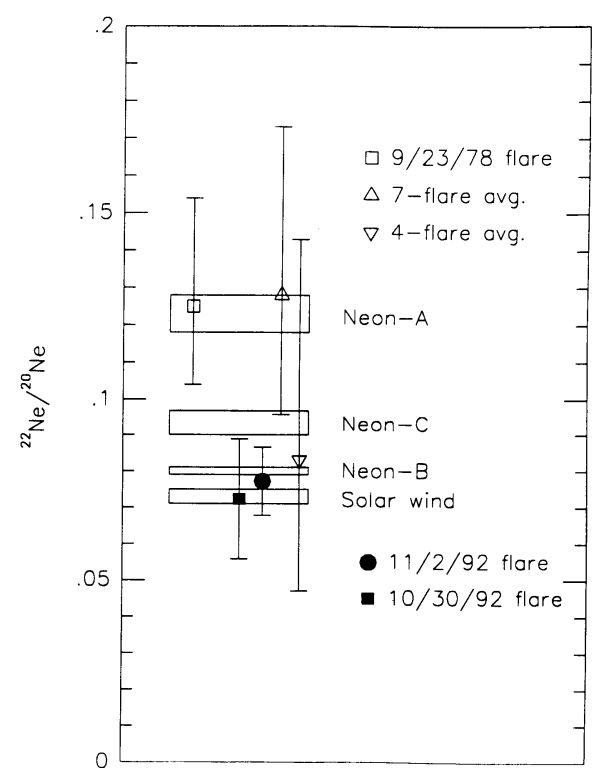

Fig. 3.-Comparison of the coronal ${ }^{22} \mathrm{Ne} /{ }^{20} \mathrm{Ne}$ ratios determined from MAST data for the 1992 SEP events with selected other solar system values. Previous SEP measurements include 11 to $51 \mathrm{MeV}$ nucleon $^{-1}$ ISEE-3 (ICE) data from the 1978 September 23 flare (Mewaldt et al. 1984), a seven-flare average of 35 to $55 \mathrm{MeV}$ nucleon ${ }^{-1}$ data from IMP-8 (Dietrich \& Simpson 1979), and a four-flare average of $\sim 14$ to $32 \mathrm{MeV}$ nucleon $^{-1}$ from the S81-1 mission (Simpson, Wefel, \& Zamow 1983), all of which have been corrected to coronal abundances as described in the text. Values for Ne-A and Ne-B are from Podosek (1978), and that for Ne-C is from Black (1983). The solar wind value is from Geiss et al. (1972). 
Cameron (1982). Also shown in Figure 3 are $\mathrm{Ne}-\mathrm{B}$, which is observed in both meteoritic and lunar material and is believed to represent directly implanted solar wind, $\mathrm{Ne}-\mathrm{C}$, which is also observed in lunar and meteoritic material and is interpreted to represent implanted SEP neon with a typical energy of $\sim 1$ $\mathrm{MeV}$ nucleon ${ }^{-1}$ (see, e.g., the review by Black 1983). Recent measurements of implanted SEP neon include ${ }^{22} \mathrm{Ne} /$ ${ }^{20} \mathrm{Ne}=0.086 \pm 0.002$ from meteorites (Rao et al. 1991) and ${ }^{22} \mathrm{Ne} /{ }^{20} \mathrm{Ne}=0.0885 \pm 0.0024$ from lunar soils (Wieler, Baur, \& Signer 1986). It is interesting to note that the ratio for the implanted SEP $\mathrm{Ne}$, which is taken to be a long-term $\left(\sim 10^{4}\right.$ $10^{5} \mathrm{yr}$ ) average, is between the extreme values observed in the 1978 and 1992 flare events. We have not applied any $Q / M$ dependent corrections to $\mathrm{Ne}-\mathrm{C}$ because of a lack of knowledge of the appropriate $\mathrm{Fe} / \mathrm{O}$ ratio. Measurements of contemporary flares (Mazur et al. 1993) suggest that this correction would be relatively small.

The difference between the previous SEP data and our new measurements suggests the possibility of flare-to-flare variations in the ${ }^{22} \mathrm{Ne} /{ }^{20} \mathrm{Ne}$ ratio that are not accounted for by our fractionation corrections based on $Q / M$. To estimate the probability of this we assume that the three ${ }^{22} \mathrm{Ne} /{ }^{20} \mathrm{Ne}$ ratios measured in the 1978 September 23 event and the two 1992 events are statistical fluctuations from a common true value. Assuming Gaussian uncertainties (given by the lower error bar for the 1978 September 23 event), the most likely true value is ${ }^{22} \mathrm{Ne} /{ }^{20} \mathrm{Ne}=0.0825$. The $\chi^{2}$ of the three measurements from this value is 4.79. The statistical significance of the result, i.e., the probability of obtaining a $\chi^{2} \geq 4.79$ from any three measurements, is $\sim 9 \%$. A common value therefore appears possible, although unlikely. However, if the common value is assumed to be that of the solar wind, then such a possibility is considerably more remote. For the statistical uncertainties associated with the ${ }^{22} \mathrm{Ne} /{ }^{20} \mathrm{Ne}$ measurement from the 1978 September 23 flare (Mewaldt, Spalding, \& Stone 1984), and assuming a true ratio equal to the solar wind value of 0.073 there is a $0.4 \%$ probability (based on Poisson statistics) of obtaining a ratio greater than or equal to the observed 0.125 . It is, of course, possible that the solar wind ratio is also affected by mass-dependent fractionation during solar wind acceleration (e.g., Geiss 1973).

The ${ }^{26} \mathrm{Mg} /{ }^{24} \mathrm{Mg}$ ratio from both of the 1992 flares is consistent with the standard solar system value $(0.139)$ of Anders \& Ebihara (1982). It is also consistent within $1 \sigma$ with the 1978 September 23 flare measurement of Mewaldt \& Stone (1989) in the energy range from 12 to $36 \mathrm{MeV}^{\text {nucleon }}{ }^{-1}$, whose value corrected to the Garrard \& Stone (1993) corona is $0.166_{-0.029}^{+0.048}$. In addition, a 10 flare average SEP value of $0.13 \pm 0.04$ (35 to $55 \mathrm{MeV}$ nucleon $^{-1}$ ) was obtained by Dietrich \& Simpson (1981). Thus, for the small sample of solar events studied to date, the substantial variability in the SEP ${ }^{22} \mathrm{Ne} /{ }^{20} \mathrm{Ne}$ abundance ratio is not evident for ${ }^{26} \mathrm{Mg} /{ }^{24} \mathrm{Mg}$, and the origin of the apparent variability in the $\mathrm{Ne}$ isotopes remains unresolved.

In summary, the new $S A M P E X$ data presented here results in a coronal ${ }^{22} \mathrm{Ne} /{ }^{20} \mathrm{Ne}$ ratio of $0.076 \pm 0.008$, considerably less than that based on previous spacecraft SEP data but in the range of the solar wind ratio and possibly Ne-C. When compared with previous SEP measurements, there is some evidence for flare-to-flare variability in ${ }^{22} \mathrm{Ne} /{ }^{20} \mathrm{Ne}$ that is greater than expected from variations in the $\mathrm{Fe} / \mathrm{O}$ ratio in the same flares, although there is currently no evidence for corresponding variability in the available ${ }^{26} \mathrm{Mg} /{ }^{24} \mathrm{Mg}$ measurements. Future work to determine additional elemental and isotopic abundances from the 1992 and other flare events will provide additional information about any such variability and may provide some insight into its possible origin.

This work was supported by NASA under contract NAS 5-30704 and grant NAGW-1919.

\section{REFERENCES}

Anders, E., \& Ebihara, M. 1982, Geochim. Cosmochim. Acta, 46, 2363

Black D. C. 1983, ApJ, 266, 889

Bochsler, P. 1989, J. Geophys. Res. 94, 2365

Breneman, H. H., \& Stone, E. C. 1985, ApJ, 299, L57

Cameron, A. G. W. 1982, in Essays in Nuclear, Astrophysics, ed. C. A. Barnes, D. D. Clayton, \& D. N. Schramm (Cambridge: Cambridge Univ. Press), 23

Cook, W. R., et al. 1993, IEEE Trans. Geoscience Remote Sensing, 31, 557

Cummings, J. R Cummings, A. C., Mewaldt, R. A., Selesnick, R. S., Stone, E. C., \& von Rosenvinge, T. T. 1993, Geophys. Res. Lett., 20, 2003

Dietrich, W. F., \& Simpson, J. A. 1979, ApJ, 231, L91

. 1981, ApJ, 245, L41

Garrard, T. L \& Stone, E. C. 1993, Proc. 23d Internat. Cosmic-Ray Conf (Calgary), 3, 384

Geiss, J. 1973, Proc. 13th Internat. Cosmic-Ray Conf. (Denver), 5, 3375

Geiss, J., Buehler, F., Cerruti, H., Eberhardt, P., \& Filleux, Ch. 1972, Apollo 16 Preliminary Science Report (NASA SP-315), 14-1

Kahler, S. W. 1993, J. Geophys. Res., 98, 5607

Luhn, A., Klecker, B., Hovestadt, D., Gloeckler, G., Ipavich, F. M., Scholer, M., Fan, C. Y., \& Fisk, L. A. 1984, Adv. Space Res., 4, 161

Mazur, J. E., Mason, G. M., Klecker, B., \& McGuire, R. E. 1993, ApJ, 404, 810

Mewaldt, R. A., Spalding, J. D., \& Stone, E. C. 1984, ApJ, 280, 892
Mewaldt, R. A., Spalding, J. D., Stone, E. C., \& Vogt, R. E. 1979, ApJ, 231, L97

Mewaldt, R. A., \& Stone, E. C. 1989, ApJ, 337, 959

Meyer, J. P. 1985, ApJS, 57, 151

1992 Adv. Space Res. in press

Parkinson, J. H. 1977, A\&A, 57, 185

Podosek, F. A. 1978, ARA\&A, 16, 293

Rao, M. N., Garrison, D. H., Bogard, D. D., Badhwar, G., \& Murali, A. V. 1991, J. Geophys. Res. 96, 321

Reames, D V 1990 , ApJS, 73, 235

Rossi, B., \& Olbert, S. 1970, Introduction to the Physics of Space (New York: McGraw-Hill)

Selesnick, R. S., Cummings, A. C., Cummings, J. R., Leske, R. A., Mewaldt, R. A. Stone, E. C., \& von Rosenvinge, T. T. 1993, Proc. 23d Internat. Cosmic Ray Conf., 3, 392

Simpson, J. A., Wefel, J. P., \& Zamow, R. 1983, Proc. 18th Internat. CosmicRay Conf. (Bangalore), 10,322

Stone, E. C. 1989, in AIP Conference Proceedings, Vol. 183, Cosmic Abundances of Matter, ed. C. J. Waddington (New York: AIP), 72

von Steiger, R., \& Geiss, J. 1989, A\&A, 225, 222

Wieler, R., Baur, H., \& Signer, P. 1986, Geochim. Cosmochim. Acta, 50, 1997 